\title{
Design of an output feedback variable structure thyristor-controlled series compensator for improving power system stability
}

\author{
Tain-Syh Luor, Yuan-Yih Hsu * \\ Department of Electrical Engineering, National Taiwan University, Room 540, No 1, Sec 4, Roosevelt Road, Taipei, Taiwan
}

Received 23 February 1998; accepted 30 March 1998

\begin{abstract}
An output feedback variable structure controller is designed for a thyristor-controlled series compensator (TCSC) in order to improve the damping characteristic of a power system. Physically measurable real power and reactive power signals near TCSC locations are used as the inputs to the variable structure controller. These input signals are employed to construct the switching hyperplane of the proposed variable structure TCSC controller. To demonstrate the effectiveness of the proposed variable structure TCSC controller, time-domain simulations are performed on a power system with a TCSC. It is concluded from the simulation results that system damping can be significantly improved by the proposed variable structure TCSC controller. (C) 1998 Elsevier Science S.A. All rights reserved.
\end{abstract}

Keywords: Thyristor-controlled series compensators; Power system stability; Variable structure control

\section{Introduction}

Due to the difficulty in construction new transmission lines, how to increase power transfer capability of existing lines using the concept of flexible ac transmission system (FACTS) received much attention in recent years $[1,2]$. Among the various FACTS controllers proposed so far, the thyristor-controlled series compensator (TCSC) [3-5] will be examined in this paper.

It is well-known that the power flow over a transmission line can be modulated through the action of variable series compensation provided by the TCSC $[1,2]$. Stability of the system will be affected as a result of the power modulation by the TCSC $[6,7]$.

In recent years, considerable attention has been placed on examining the effect of TCSC on power system stability [7-16]. In [7], a control signal which is proportional to the difference between the angular velocities of the two machines at the two ends of the lines was proposed to modulate TCSC reactance. The eigenvalues of the oscillation mode were computed and the effects of line loading and load characteristics on sys-

\footnotetext{
* Corresponding author.
}

tem damping was evaluated. In [8], a state feedback controller was designed for the TCSC by using a pole placement technique. The desired closed-loop poles were reached by a feedback gain matrix. All system states were required by the feedback controller.

In a recent work by Chang and Chow [9], a time optimal control strategy was developed for the TCSC. A performance index of time was minimized to reach the goal of minimal time control. In [10], a fuzzy logic control scheme was proposed for a TCSC to enhance the transient stability of a power system. The series impedance of the TCSC was adjusted according to a fuzzy assignment table, which was established based on machine rotor angle $\delta$ and the magnitude of the speed deviation $|\Delta \omega|$. Dynamic responses of the system with a combination of a conventional power system stabilizer (PSS) and the proposed fuzzy logic controller were investigated. Recently, Wang et al. [11] presented a variable structure controller for the TCSC. The original fourth-order model was first simplified to a second-order system Then, the switching hyperplane was constructed using generator rotor angle and speed.

Note that the generator rotor angle, angular speed and other variables were employed as the inputs to the 
controller in [7-11]. In practice, the TCSC is normally installed at a substation far from the generating plant. Therefore, the above mentioned variables of the generator are not readily available at the TCSC substation. Usually, only real and reactive power flows, and voltage and current measurements are available at the location of the TCSC. To estimate the required phase/speed states from the measured real power flow at the location of TCSC, a signal conditioning system comprising reset filters and integrators was developed in [12]. A fuzzy logic controller for the TCSC was then designed based on the estimated phase and speed. The effectiveness of the fuzzy logic TCSC was compared with a fuzzy logic PSS. In Refs. [13,14], a pair of remote voltages were synthesized using the voltages and currents measured at the terminals of a TCSC and the Thevenin impedances. Then, the synthesized angle signal was computed as the difference between the phase angles of the two synthesized voltages. This angle signal was employed for damping control.

In contrast to the works in Refs. [12-14], which required a signal conditioning system or voltage synthesizer in order to generate the inputs to the TCSC, de Mello [15] examined the control of TCSC reactance using the rate of change of the measured through-power in series of branches as the controller input. Analysis and simulation results indicated that the damping has been improved by the proposed rate feedback controller.

In this work, an output feedback variable structure controller (VSC) is proposed to modulate the series impedance of the TCSC. In contrast to the work in [11], which employs generator rotor angle and shaft speed, which are not readily available at the location of the TCSC, as feedback variables, only the real power and reactive power measured at the terminal of the TCSC are used as controller inputs. A major feature of the variable structure controller is that dynamic performance of the system can be significantly improved when the VSC is operated in the so-called sliding mode motion [16-20]. In addition, the variable structure controllers are quite insensitive to system parameter variations.

To demonstrate the effectiveness of the proposed output feedback VSC, computer simulations are performed on a power system with TCSC. It is concluded from the simulation results that damping charac- teristics of the system can be improved by the proposed VSC.

\section{System model}

The system under study is a synchronous generator connected to a large power system through a transmission line equipped with a TCSC, as shown in Fig. 1. The variable series compensation, $D_{x}$, provided by the TCSC is controlled by a TCSC controller. A variable structure TCSC controller will be designed in the next section to improve the damping of system oscillations.

Fig. 2 depicts the block diagram of the excitation system, which can be described by the following differential equations:

$$
\begin{aligned}
& T_{\mathrm{A}} \dot{E}_{\mathrm{FD}}=K_{\mathrm{A}}\left(V_{\mathrm{ref}}-V_{t}\right)-E_{\mathrm{FD}} \\
& T_{\mathrm{F}} \dot{V}_{\mathrm{S}}=K_{\mathrm{F}} \dot{E}_{\mathrm{FD}}-V_{\mathrm{S}}
\end{aligned}
$$

\subsection{The complete model of synchronous generator}

To simulate the dynamic responses of the system under disturbance, a complete nonlinear generator model [21] is used. The model is described by a set of seven nonlinear differential equations of the form

$\dot{\boldsymbol{X}}^{\prime}(t)=f\left(\boldsymbol{X}^{\prime}(t), u^{\prime}(t)\right)$,

where $\boldsymbol{X}^{\prime}(t)=\left[i_{d}, i_{q}, i_{F}, i_{D}, i_{Q}, \omega, \delta\right]^{T}$ is the state vector and $u^{\prime}$ is the control signal. Details for the seven differential equations can be found in [21].

To evaluate the eigenvalues of the system, Eq. (3) is linearized around a nominal operating point to get

$\dot{\boldsymbol{X}}^{\prime}(t)=A^{\prime} \boldsymbol{X}^{\prime}(t)+B^{\prime} u^{\prime}(t)$

The eigenvalues of the generator equipped with the excitation system in Fig. 2 are summarized in Table 1.

It is observed from Table 1 that the electromechanical mode for the open-loop system (without TCSC, $D_{x}=0$ ), which are characterized by the eigenvalues $0.13 \pm j 9.95$, is poorly damped. If an adjustable series reactance, as described in figure 7 of [15] is implemented, the damping of low frequency electromechanical mode oscillation will be improved, as evidenced by the eigenvalues $-2.59 \pm$ j11.56.

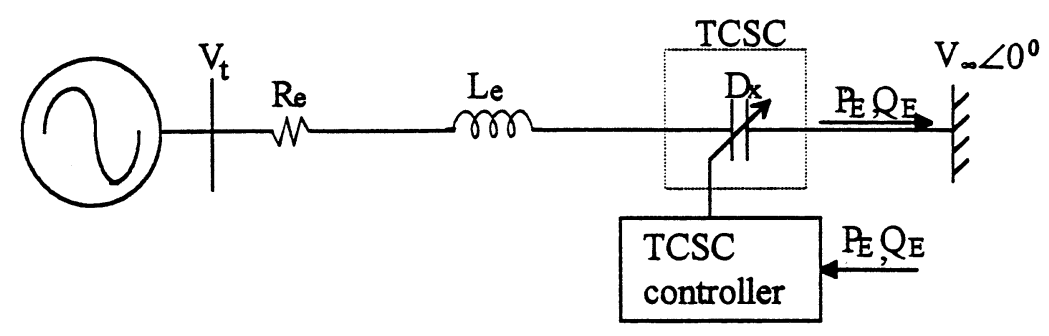

Fig. 1. System configuration for a synchronous machine connected to a large power system through a transmission line with a TCSC. 


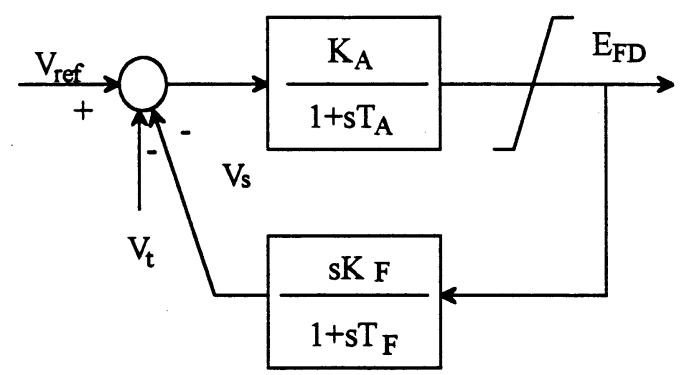

Fig. 2. The excitation system.

Table 1

System eigenvalues at $P=1.0, Q=0.62$ and $V_{t}=1.0$

\begin{tabular}{lc}
\hline Open-loop system $\left(D_{x}=0\right)$ & System with TCSC in [15] \\
\hline$-0.7 \pm j 376.9$ & $-51.9 \pm j 292.7$ \\
$-6.8 \pm j 21.6$ & $-51.5 \pm j 6.14$ \\
$0.13 \pm j 9.95$ & $-5.25 \pm j 18.95$ \\
-47.9 & $-2.59 \pm j 11.56$ \\
-44.7 & -378.9 \\
-1.0 & -1.0 \\
& -15.9 \\
\hline
\end{tabular}

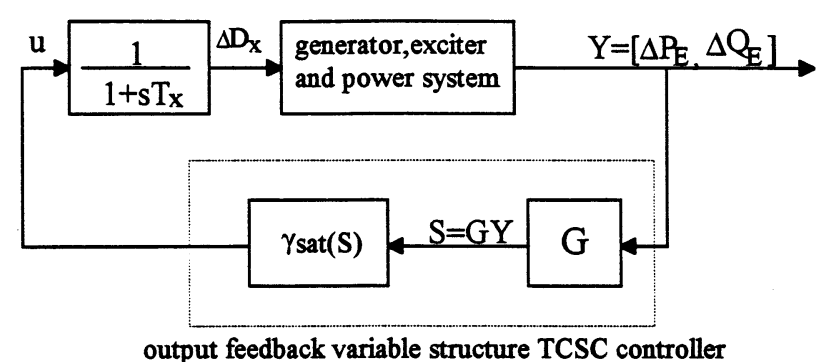

Fig. 3. Block diagram of the power system with the proposed output feedback variable structure TCSC controller.

\subsection{Simplified model of the synchronous generator}

In the design of a variable structure TCSC controller, the synchronous generator is described by a simplified third-order linear model described below.

$$
\Delta \dot{\delta}=\Delta \omega
$$

$\tau_{j} \Delta \dot{\omega}=\Delta T_{m}-D \Delta \omega$

$$
-\left(I_{d 0} \Delta E_{d}^{\prime}+I_{q 0} E_{q}^{\prime}+E_{d 0}^{\prime} \Delta I_{d}+E_{q 0}^{\prime} \Delta I_{q}\right)
$$

$\tau_{d 0}^{\prime} \Delta E_{q}^{\prime}=\Delta E_{\mathrm{FD}}-\Delta E_{q}^{\prime}-\left(x_{d}-x_{d}^{\prime}\right) \Delta I_{d}$

$\left[\begin{array}{l}\Delta E_{q}^{\prime} \\ \Delta E_{d}^{\prime}\end{array}\right]=\left[\begin{array}{l}\Delta V_{q} \\ \Delta V_{d}\end{array}\right]+\left[\begin{array}{cc}-x_{d}^{\prime} & 0 \\ 0 & x_{q}^{\prime}\end{array}\right]\left[\begin{array}{l}\Delta I_{d} \\ \Delta I_{q}\end{array}\right]$

The system voltage equations are written as

$$
\begin{aligned}
v_{d}= & -\sqrt{3} V_{\infty} \sin \delta+\left(L_{e}-D_{x} / \omega_{R}\right) i_{d} \\
& +\omega\left(L_{e}-D_{x} / \omega_{R}\right) i_{q}
\end{aligned}
$$

$v_{q}=\sqrt{3} V_{\infty} \cos \delta+\left(L_{e}-D_{x} / \omega_{R}\right) i_{q}-\omega\left(L_{e}-D_{x} / \omega_{R}\right) i_{d}$

(10)
Linearizing Eqs. (9) and (10) and substituting into Eq. (8), one gets

$$
\begin{aligned}
& \Delta I_{d}=K_{1} \Delta \delta+K_{2} \Delta E_{q}^{\prime}+K_{3} \Delta D_{x} \\
& \Delta I_{q}=K_{4} \Delta \delta+K_{5} \Delta D_{x}
\end{aligned}
$$

where

$$
\begin{array}{ll}
K_{1}=\frac{-V_{\infty} \sin \delta_{0}}{x_{d}^{\prime}+x_{e}-D_{x 0}}, & K_{4}=\frac{V_{\infty} \cos \delta_{0}}{x_{q}^{\prime}+x_{e}-D_{x 0}}, \\
K_{2}=\frac{-1}{x_{d}^{\prime}+x_{e}-D_{x 0}}, & K_{5}=\frac{V_{\infty} \sin \delta_{0}}{\left(x_{q}^{\prime}+x_{e}-D_{x 0}\right)^{2}}, \\
K_{3}=\frac{V_{\infty} \cos \delta_{0}-E_{q 0}^{\prime}}{\left(x_{d}^{\prime}+x_{e}-D_{x 0}\right)^{2}} &
\end{array}
$$

As shown in Fig. 1, real and reactive power flows over the transmission line, $P_{E}$ and $Q_{E}$, are taken as the output feedback signals to the TCSC controller. In other words, the TCSC controller uses measured system outputs $P_{E}$ and $Q_{E}$ as its inputs. These controller inputs can be expressed as

$$
\begin{aligned}
& P_{E}=V_{\infty d} I_{d}+V_{\infty q} I_{q} \\
& Q_{E}=V_{\infty d} I_{q}-V_{\infty q} I_{d}
\end{aligned}
$$

By substituting Eqs. (11) and (12) into Eqs. (13) and (14), the system outputs can be expressed as functions of state variables $\Delta \delta$ and $\Delta E_{q}^{\prime}$ and controller output $\Delta D_{x}$

$\Delta P_{E}=C_{1} \Delta \delta+C_{2} \Delta D x+C_{3} \Delta E_{q}^{\prime}$

$\Delta Q_{E}=C_{4} \Delta \delta+C_{5} \Delta D_{x}+C_{6} \Delta E_{q}^{\prime}$

Fig. 3 depicts the block diagram of generator, exciter and power system and the output feedback variable structure TCSC controller.

Thus, the simplified model of the generator, exciter, and the power system equipped with a TCSC can be described by the following equations.

$\dot{\boldsymbol{X}}(t)=A \boldsymbol{X}(t)+B u(t)$

$\boldsymbol{Y}(t)=C \boldsymbol{X}(t)$

where $\boldsymbol{X}(t)=\left[\Delta \delta, \Delta \omega, \Delta E_{q}^{\prime}, \Delta E_{\mathrm{FD}}, \Delta V_{\mathrm{S}}, \Delta D_{x}\right]^{T}$ is the state vector, and $Y(t)=\left[\Delta P_{E}, \Delta Q_{E}\right]^{T}$ is the output vector, and $u$ is the control signal. Details on the design of the variable structure TCSC controller will be described in the next section.

\section{Design of the output feedback variable structure TCSC controller}

For the system in Eqs. (17) and (18), the purpose of variable structure TCSC controller design is to find a proper control $u$ such that the system is stabilized robustly. In other words, good damping effect must be provided by the control $u$ over a wide range of operat- 
ing conditions. In variable structure control, the control $u$ is of the form [22-24]

$u=\left\{\begin{array}{lll}u^{+}(\boldsymbol{Y}) & \text { if } & S(\boldsymbol{Y})>0 \\ u^{-}(\boldsymbol{Y}) & \text { if } & S(\boldsymbol{Y})<0\end{array}\right.$

where

$S(\boldsymbol{Y})=G \boldsymbol{Y}$

is the switching function. The so-called sliding mode motion [16] takes place on the sliding hyperplane,

$S(\boldsymbol{Y})=0$.

The first step in variable structure controller design is to determine a proper sliding hyperplane. Using the method of equivalent control [16], the equation of motion in the sliding mode for the system in Eq. (17) is described as follows:

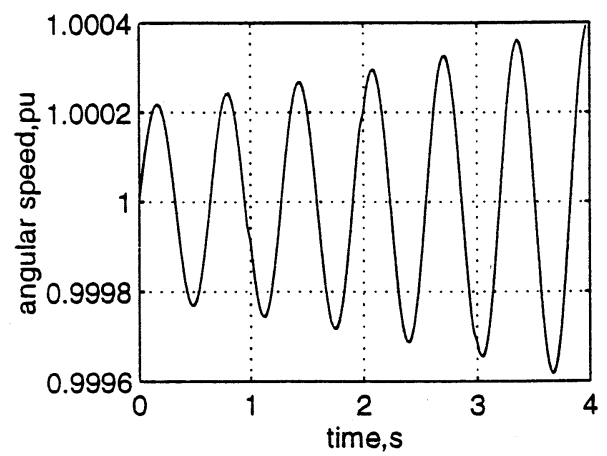

$$
\dot{S}(t)=G \dot{\boldsymbol{Y}}(t)=G C \dot{\boldsymbol{X}}(t)=G C A \boldsymbol{X}(t)+G C B u(t)
$$

Since the equivalent control satisfies the condition $\dot{S}=$ 0 , we have the equivalent control

$u_{\mathrm{eq}}(t)=-(G C B)^{-1} G C A X(t)$

Then Eq. (17) reduces to

$$
\dot{X}(t)=\left[A-B(G C B)^{-1} G C A\right] \boldsymbol{X}(t)=\bar{A} \boldsymbol{X}(t)
$$

Note that the switching function $S(\boldsymbol{Y})$ should be chosen such that the sliding mode equation (Eq. (24)) is stabilized under the constraint that $G C B$ is invertible. It is also noted that Eq. (21) must be satisfied in the sliding mode. Since the number of outputs (two in the present work) exceed the number of control signal (one in this work), some freedom remains in choosing the sliding surface [23].

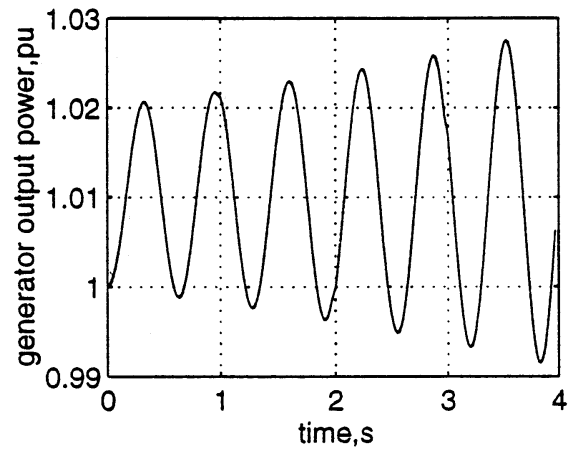

Fig. 4. Dynamic responses of the system without TCSC $(P=1.0, Q=0.62)$.
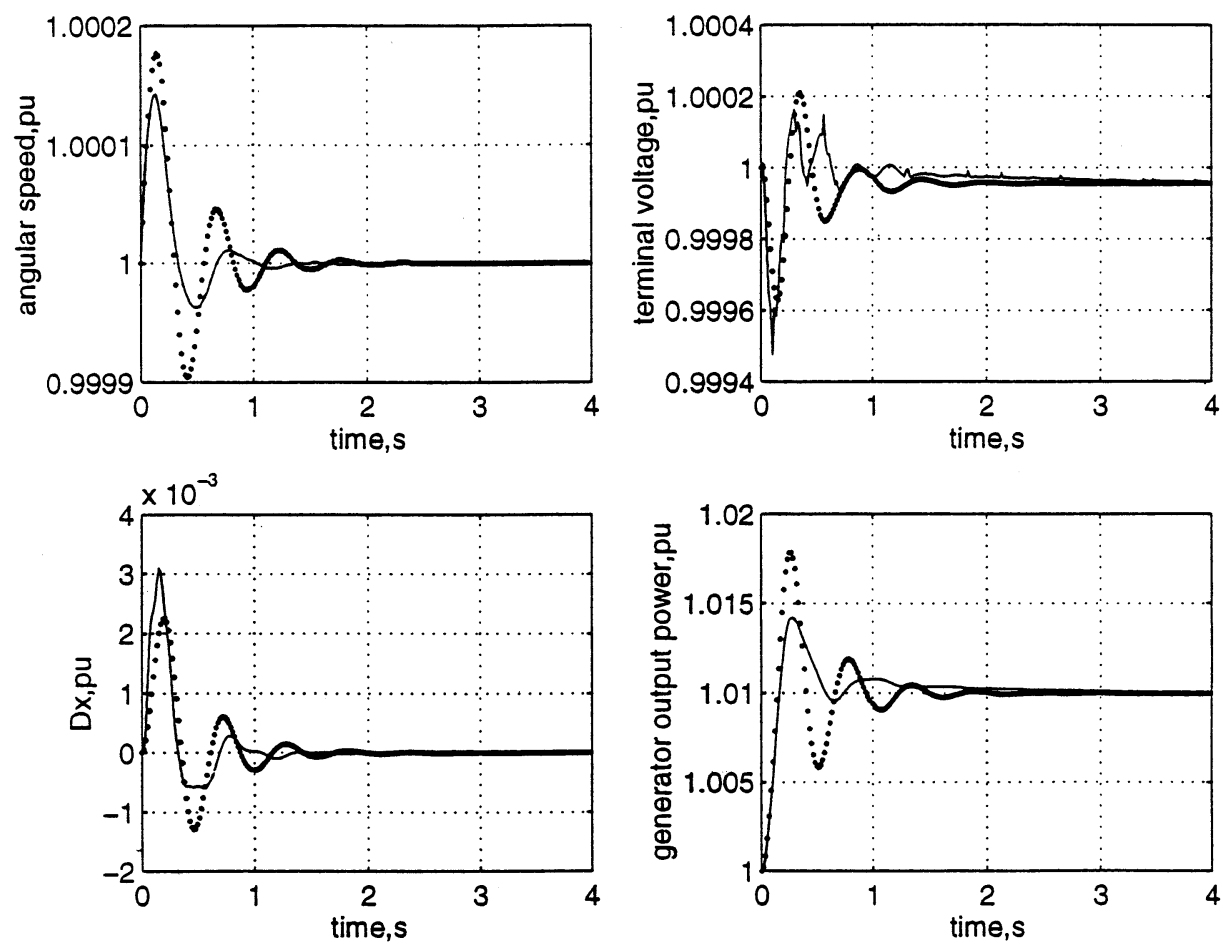

TCSC with variable structure controller

TCSC with rate feedback controller [15]

Fig. 5. Dynamic responses of the system with TCSC $(P=1.0, Q=0.62)$. 

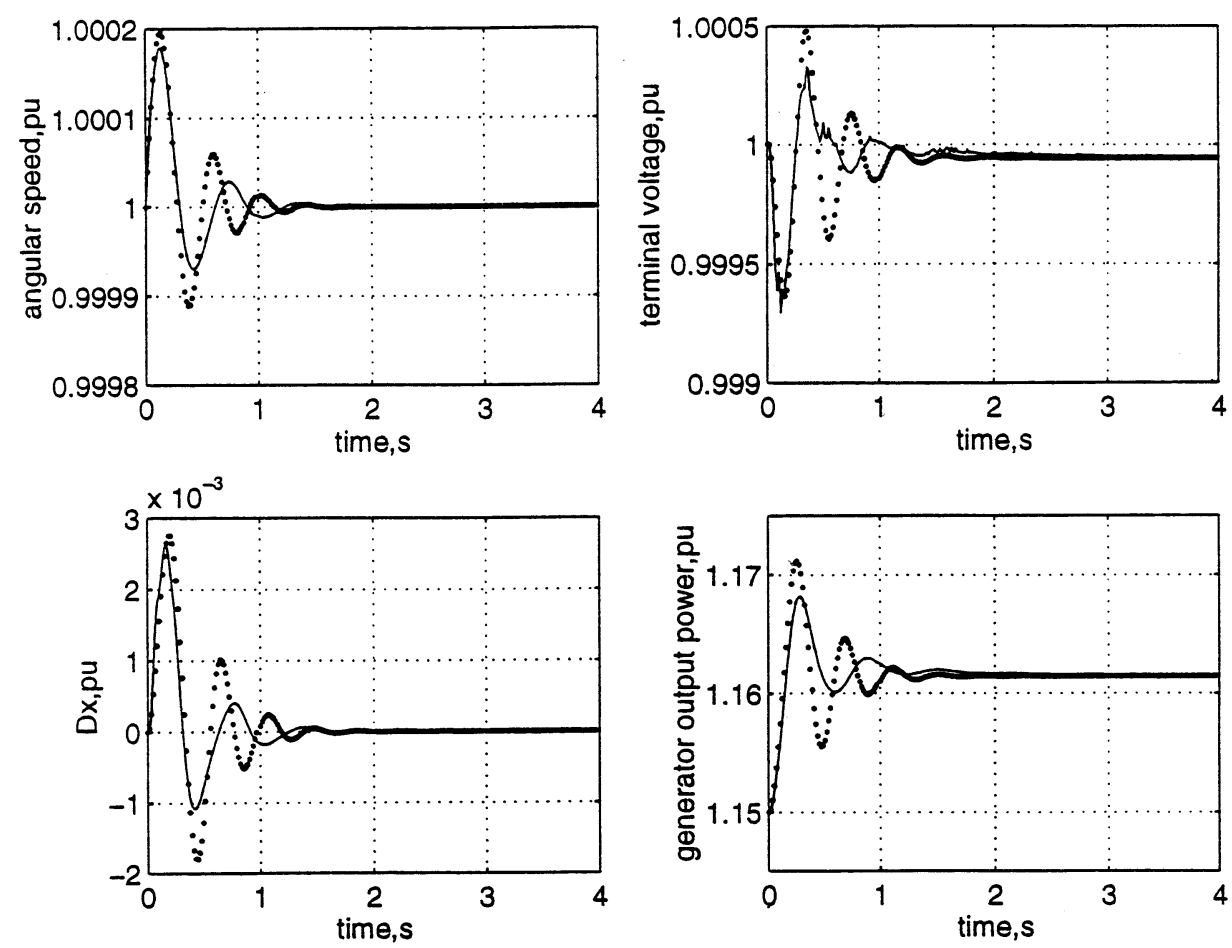

TCSC with variable structure controller TCSC with rate feedback controller [15]

Fig. 6. Dynamic responses of the system with TCSC $(P=1.15, Q=0.71)$.

In order for the system to reach the sliding hyperplane, the following reaching condition [16] must be satisfied

$S(\boldsymbol{Y}) \dot{S}(\boldsymbol{Y})<0$

Let the control $u$ in Eq. (19) be

$u=\gamma \operatorname{sgn}(S)$

where $\gamma$ is a constant gain that will be determined by using the equivalent control $u_{\text {eq }}$.

Suppose that the system state is not on the sliding surface $S=0$ but is close to it. Then we have, from Eqs. (22) and (23)

$\dot{S}(t)=G C A X(t)+G C B u(t)=G C B\left(u(t)-u_{\mathrm{eq}}(t)\right)$

To satisfy the reaching condition in Eq. (25), we get

$\gamma<\max \left|u_{\text {eq }}\right| \quad$ if $\quad(G C B)<0$

$\gamma>\max \left|u_{\text {eq }}\right| \quad$ if $\quad(G C B)>0$

Thus, the value max $\left|u_{\mathrm{eq}}\right|$ should be estimated in order to obtain a proper constant $\gamma$. This is usually done by applying the Schwartz inequality to Eq. (23) to have $\left|u_{\mathrm{eq}}\right|<\left\|(G C B)^{-1} G C A\right\|\|x\|[24]$.

To avoid a chattering problem in sliding mode motion, the sgn function in Eq. (26) can be replaced by a sat function [22-24] $u=\gamma \operatorname{sgn}(S)=\left\{\begin{array}{ccc}\gamma \operatorname{sgn}(S) & \text { if } & S>|\varepsilon| \\ \frac{\gamma}{\varepsilon} S & \text { if } & S \leq|\varepsilon|\end{array}\right.$

where $\varepsilon$ is a small constant.

\section{Example}

To demonstrate the effectiveness of the proposed variable structure TCSC controller, dynamic responses of the system subject to a 0.01 pu step change in mechanical torque $\Delta T_{m}$ are examined. Note that the complete nonlinear model of synchronous generator as described in Section 2.1 is employed in the computer simulation. Fig. 4 illustrates the response curves of the system without TCSC and TCSC controller under the operating condition of $P=1.0 \mathrm{pu}, Q=0.62 \mathrm{pu}$ and $V_{t}=1.0 \mathrm{pu}$.

For the switching hyperplane in the variable structure controller, we select $G=[70,-10]$ such that the eigenvalues of sliding mode equation (Eq. (24)) are located in the left complex plane. We choose $\gamma=0.3$ to satisfy Eq. (28).

The dynamic response curves for the system with TCSC and the proposed variable structure TCSC controller are shown in Figs. 5 and 6 for the operating conditions of $P=1.0 \mathrm{pu}, Q=0.62 \mathrm{pu}$ and $P=1.15 \mathrm{pu}$, $Q=0.71 \mathrm{pu}$, respectively. 


\section{Discussions}

From the results in Figs. 4-6, the following observations are in order.

(1) As evidenced by the response curves in Fig. 4, the system without TCSC is unstable. This is as expected since the electromechanical mode (characterized by the eigenvalues $0.13 \pm j 9.95$ in Table 1) has negative damping.

(2) The unstable eigenvalues $0.13 \pm j 9.95$ will be shifted to $-2.59 \pm j 11.56$ (Table 1) as the TCSC with a rate feedback control signal in [15] is added to the system. Improvement in system damping is expected in this case. In fact, an observation of the response curves in Figs. 5 and 6 for the TCSC with rate feedback controller in [15] indicates that the oscillation mode has been stabilized.

(3) On comparing the response curves from the rate feedback controller in [15] with those from the proposed variable structure controller, it is observed that the variable structure controller yields better system dynamic performance than the rate feedback controller.

(4) An observation of the response curves in Figs. 5 and 6 reveals that the variable structure TCSC controller can provide good damping characteristics even when the operating condition is changed.

(5) Smooth voltage profile can be maintained when the TCSC controller is employed to stabilize the electromechanical mode. This is a major advantage of TCSC controller over power system stabilizer $[20,21]$ which often causes relatively greater voltage deviations when used to stabilize oscillations.

\section{Conclusions}

An output feedback variable structure TCSC controller has been designed to improve the dynamic performance of a power system. A main feature of the proposed variable structure controller is that only physically measurable real power and reactive power signals are used as the controller inputs. This makes the proposed variable structure controller relatively easy for practical implementation. In addition, the dynamic performance of the system is rather insensitive to variations in operating conditions and plant parameters when the system is operated in the sliding mode. Simulation results indicate that the variable structure TCSC controller provides an effective means for improving the damping characteristic of the power system

\section{Acknowledgements}

Financial support given to this work by the National Science Council of ROC under contract number NSC85-2213-E002-069 is appreciated.

\section{References}

[1] L. Gyugyi, Unified power-flow control concept for flexible ac transmission system, IEE Proc. C 139 (1992) 323-331.

[2] EPRI, Flexible ac transmission system (FACTS): Scoping study, 1991.

[3] A. Ghosh, G. Ledwich, Modeling and control of thyristor-controlled series compensators', IEE Proc. C 142 (1995) 297-304.

[4] J.J. Paserba, N.W. Miller, E.V. Larsen, R.J. Piwko, A thyristor-controlled series compensation model for power system stability analysis, IEEE Trans. PWRD 10 (1995) 1471-1478.

[5] S.G. Jalali, R.H. Lasseter, L. Dobson, Dynamic response of a thyristor-controlled switched capacitor, IEEE Trans. PWRD 9 (1994) 609-1615.

[6] L. Angquist, B. Lundin, J. Samuelsson, Power oscillation damping using controlled reactive power compensation-a comparison between series and shunt approaches, IEEE Trans. PWRS 8 (1993) 687-698.

[7] M. Noroozian, G. Andersson, Damping of power system oscillations by use of controllable components, IEEE Trans. PWRD 9 (1994) 2046-2053.

[8] X.R. Chen, N.C. Pahalawaththa, U.D. Annakkage, C.S. Kumble, Controlled series compensation for improving the stability of multi-machine power systems, IEE Proc. C 142 (1995) 361366.

[9] J. Chang and J.H. Chow, Time-optimal series capacitor control for damping inter-area modes in interconnected power systems, Paper 96WM 262-6 PWRS, IEEE/PES Winter Meeting, 1996.

[10] T.T. Lie, G.B. Shrestha, A. Ghosh, Design and application of a fuzzy logic control scheme for transient stability enhancement in power systems, Electric Power Syst. Res. 3 (1995) $17-23$.

[11] Y. Wang, R.R. Mohler, R. Spee, W. Mittelstadt, Variablestructure FACTS controllers for power system transient stability, IEEE Trans. PWRS 7 (1992) 307-313.

[12] H. Hiyama, M. Mishiro, H. Kihara, Coordinated fuzzy logic control for series capacitor modules and PSS to enhance stability of power system', IEEE Trans. PWRD 10 (1995) 10981104.

[13] P.S. Dolan, J.R. Smith, W. Mittelstadt, A study of TCSC optimal damping control parameters for different operation conditions, IEEE Trans. PWRS 10 (1995) 1972-1977.

[14] E.V. Larsen, J.J. Sanchez-Gasca and J.H. Chow, Concepts for design of FACTS controller to damp power swings, Paper 94SM 532-2 PWRS, IEEE/PES Summer Meeting, 1994.

[15] F.P. De Mello, Exploratory concepts on control of variable series compensation in transmission systems to improve damping of intermachine/system oscillators, IEEE Trans. PWRS 9 (1994) 102-108.

[16] V.I. Utkin, Variable structure systems with sliding modes, IEEE Trans. AC 22 (1977) 212-222.

[17] V.I. Utkin, K.D. Yang, Methods for constructing discontinuity plants in multidimensional variable structure systems, Automation Remote Control 39 (1978) 1466-1470.

[18] W.C. Chan, Y.Y. Hsu, An optimal variable structure stabilizer for power system stabilization, IEEE Trans. PAS 102 (1983) $1738-1746$.

[19] W.C. Chen, Y.Y. Hsu, Automatic generation control of interconnected power system using variable-structure controllers, IEE Proc. C 128 (1981) 269-279.

[20] M.L. Kothari, J. Nanda, K. Bhattacharya, Design of variable structure power system stabilizers with desired eigenvalues in the sliding mode, IEE Proc. C 140 (1993) 263-268. 
[21] P.M. Anderson, A.A. Fouad, Power System Control and Stability, Iowa State University Press, Ames, IA, 1977.

[22] P.K. Dash, S. Mishra, A.C. Liew, Fuzzy logic based VAR stabilizer for power system control, IEE Proc. C 142 (1995) $618-624$.
[23] B.S. Heck, A.A. Ferri, Application of output feedback to variable structure systems, J. Guidence 12 (1989) 932-935.

[24] J.Y. Hung, R.M. Nelms, P.B. Stevenson, An output feedback sliding mode speed regulator for dc drives, IEEE Trans. IA 30 (1994) 691-698. 